\title{
Studying Radio-Loud AGN with eROSITA
}

\author{
Christoph GROSSBERGER ${ }^{* 1,2}$, Christian Schmid ${ }^{1}$, Thorsten Brand ${ }^{1}$, Ingo \\ Kreykenbohm $^{1}$, Jörn Wilms ${ }^{1}$, Matthias Kadler ${ }^{2}$, Michael Wille ${ }^{1}$ \\ ${ }^{1}$ Dr. Karl Remeis-Observatory \& ECAP, Sternwartstr. 7, 96049 Bamberg, Germany \\ ${ }^{2}$ Institut für Theoretische Physik und Astrophysik, Emil-Fischer Str. 31, 97074 Würzburg, \\ Germany \\ E-mail: christoph.grossbergeresternwarte.uni-erlangen.de
}

We present a study of the detection and monitoring capabilities of radio-loud Active Galactic Nuclei (AGN) with the future X-ray telescope eROSITA on-board the Russian mission Spektrum Roentgen-Gamma. The study is based on a simulation of a full 4-year all-sky survey and pointed observations. Overall up to 3 million AGN are expected to be detected by the eROSITA instrument.

11th European VLBI Network Symposium \& Users Meeting

9-12 October 2012

Bordeaux (France)

\footnotetext{
* Speaker.
} 


\section{EROSITA}

eROSITA (extended ROentgen Survey with an Imaging Telescope Array) is the primary instrument on board the Russian Spectrum-Roentgen-Gamma (SRG) mission [5-9] to be launched in 2014. The construction and development of the eROSITA instrument (see Figure 1) is under the leadership of the Max-Planck-Institute for extraterrestrial Physics (MPE). Its mirror system consists of 7 individual Wolter-type telescopes with an on axis angular resolution of about 15 arcsec at $1.5 \mathrm{keV}$. For detailed technical information about the instrument and the mission refer to the eROSITA Science Book [4].

The main goal of eROSITA is the detection of a large sample of galaxy clusters ( $\sim 10^{5}$ objects) to address the questions of cosmological models including Dark Energy and the study of the large scale structure in the Universe [4]. Therefore a deep all-sky survey of the X-ray sky $(0.2 \mathrm{keV}-$ $10 \mathrm{keV}$ ) will be performed in the first 4 years. Followed by a 3.5 years long period of pointed observations with open access to the astrophysical community by regular announcement of opportunities. The survey will be performed in an orbit around the second Lagrangian point L2 of the Sun-Earth system to avoid heat radiation from Earth. Furthermore L2 allows to perform long and stable observations [4]. The survey itself will be done by drift scan observations and is flux limited to at least one order of magnitude lower than the ROSAT All Sky Survey. An estimate of the sky coverage in equatorial coordinates is shown in Figure 2, showing that every position of the sky is observed at least 8 times while the ecliptic poles (overlap region of the individual scans) will be observed more than a hundred times.

Besides the main goal of the mission, the eROSITA all-sky survey is expected to detect more than 3 million Active Galactic Nuclei (AGN) with a median redshift of about 1 [3] making eROSITA an important instrument to study and monitor these kind of objects. Moreover this sample of AGN offers the unique opportunity to study the evolution of supermassive black holes.

In this paper, we present a simulated AGN distribution of one of the ecliptic poles after a full 4 year survey.

\section{SIMPUT}

The SIMulation inPUT (SIMPUT) ${ }^{1}$ file format can be used to define sources for input in simulations of astronomical observations. Each SIMPUT source file contains a catalog with one or multiple sources, which are described by specific properties such as position, brightness, energy spectrum, as well as optional characteristics such as time variability, polarization, and spatial extent. The file format defines a common basis to exchange data between different software packages and scientific groups. It was developed in particular for the simulation of X-ray telescopes, but can also be used in different wavelength domains.

\footnotetext{
${ }^{1}$ http://hea-www.harvard.edu/HEASARC/formats/simput-1.0.0.pdf
} 


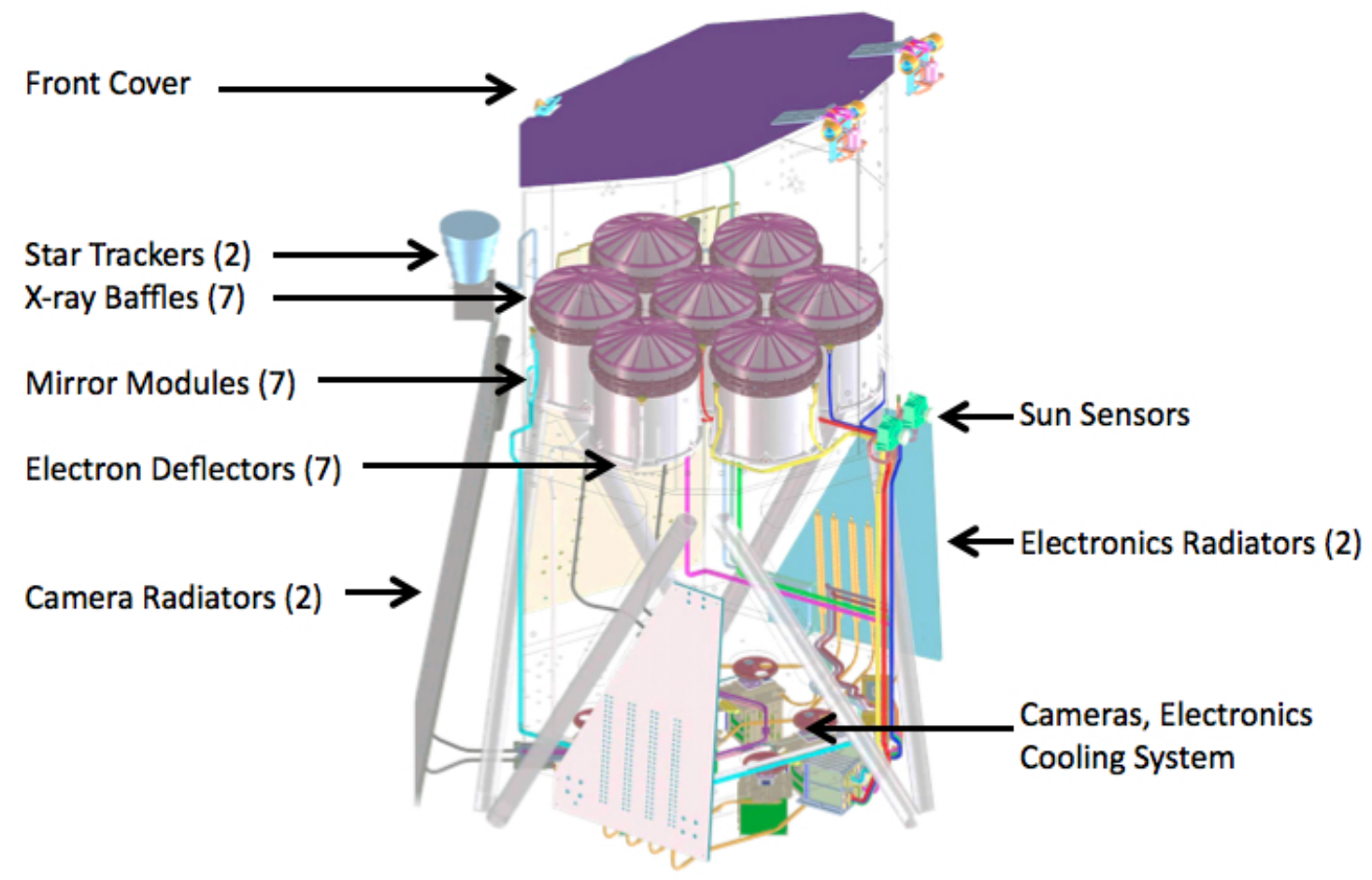

Figure 1: The eROSITA instrument [4].

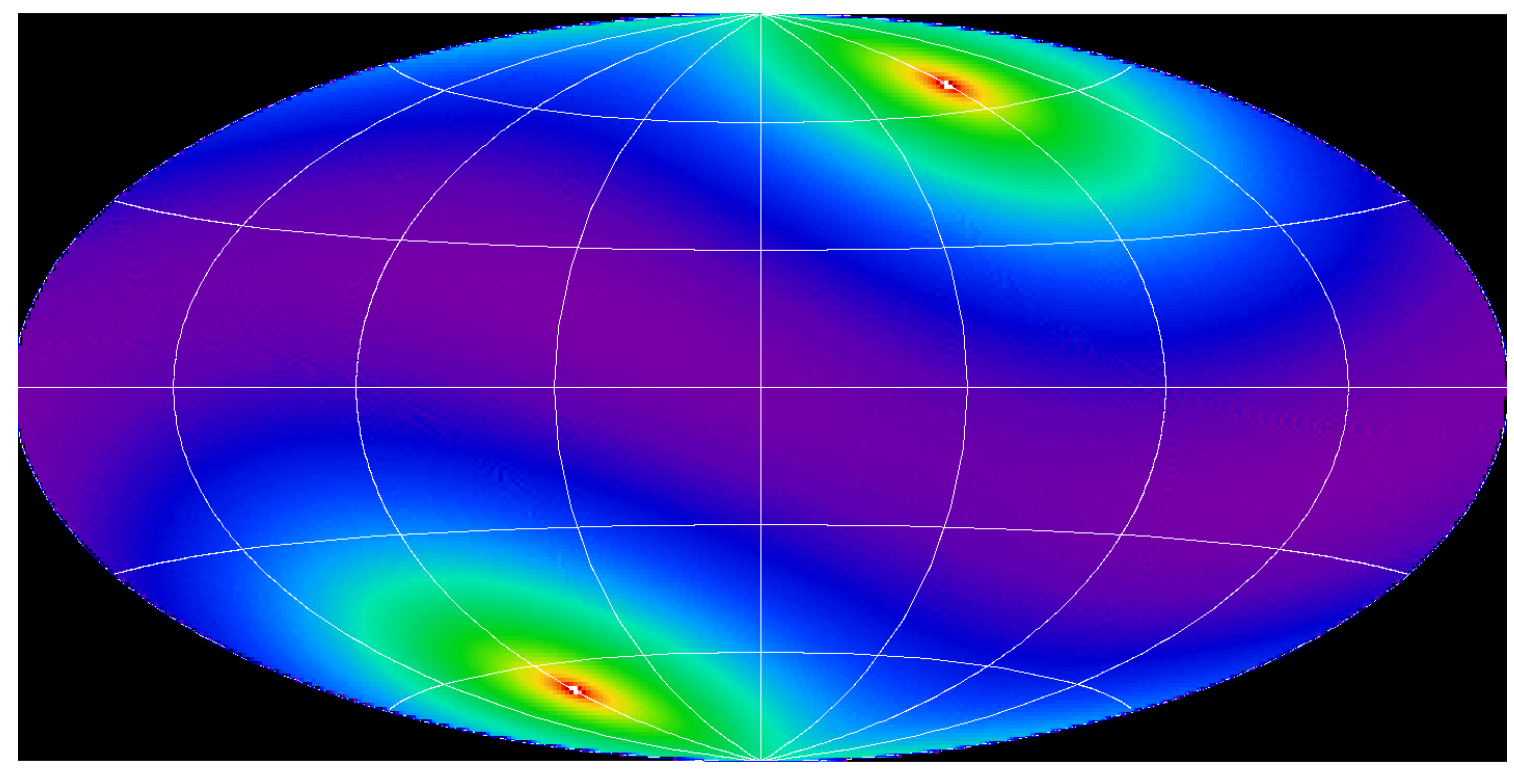

10

13

20

35

232

457

Figure 2: Cadence map - showing the number of daily visits of eROSITA on the celestial sphere in equatorial coordinates[4]. Each visit consists of $\sim 6$ overlapping scans with an exposure of $\sim 30 \mathrm{~s}$. 


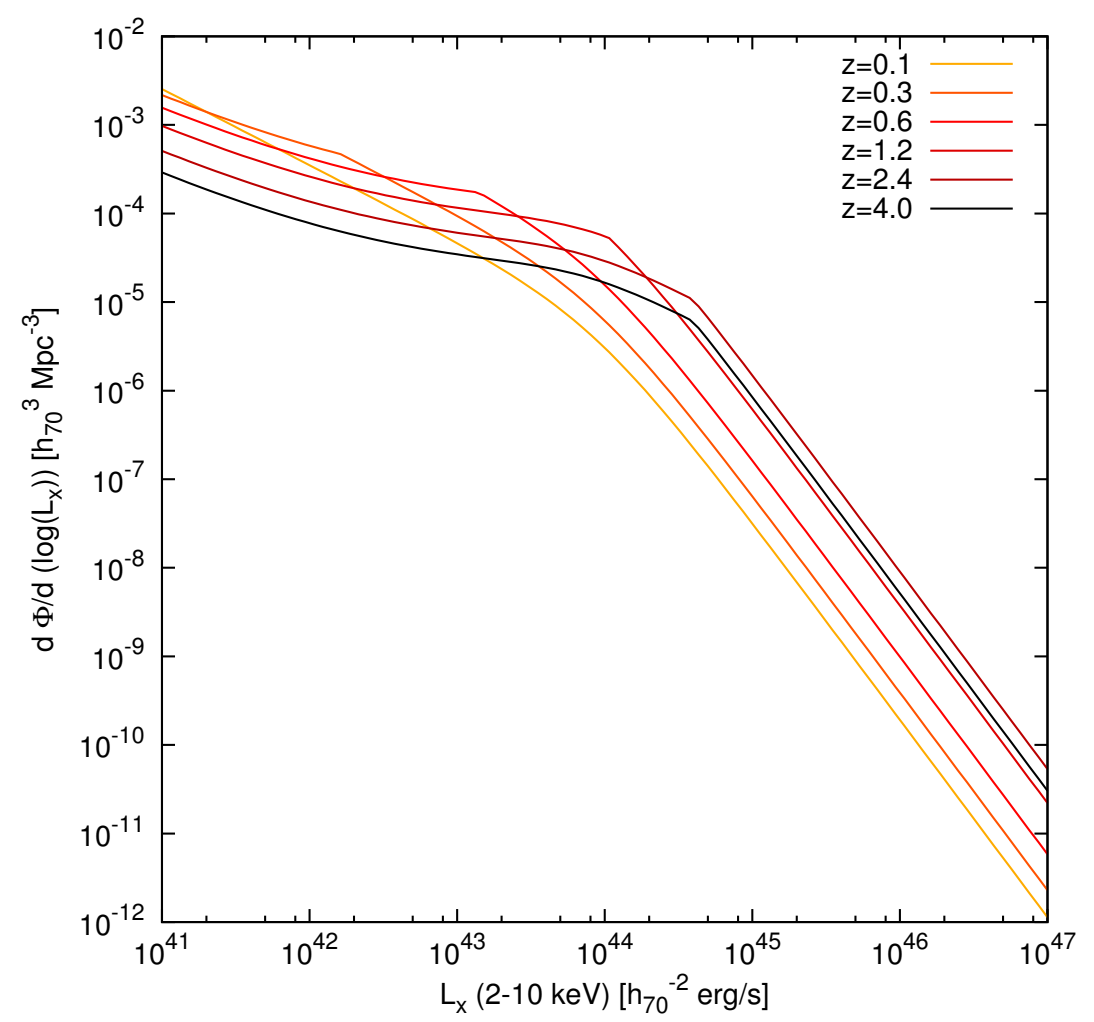

Figure 3: X-Ray Luminosity Function describing the number density of AGN in relation to their intrinsic luminosity for different redshifts.

\section{Simulating AGN}

As a first step, a SIMPUT catalog of simulated positions, spectra, and fluxes of AGN was created. Therefore, the X-Ray Luminosity function (XLF) [12] (see Figure 3) was evaluated to a redshift of $\mathrm{z}=4$ to determine the number of sources per volume and intrinsic luminosity at a given redshift. To calculate the total number of sources, the cosmological parameters were set to $H_{0}=70 \mathrm{~km} \mathrm{~s}^{-1} \mathrm{Mpc}^{-1}, \Omega_{m}=0.3, \Omega_{\Lambda}=0.7$ and $\Omega_{k}=0$. In a Monte Carlo approach, the sources were distributed uniformly across the sky and the spectral properties absorption and photon index for each AGN were assigned. The photon index follows a Gaussian distribution with a mean of 1.9 and a $\sigma_{\Gamma} 0.2$ [2]. The spectral model consists of a powerlaw with an exponential cutoff complimented by an absorption and reflection component as well as an iron line[1]. Taking these characteristics into account, the apparent flux for each AGN was calculated.

\section{Simulating a polar field}

Based on our artificial AGN catalog and a model of the detector background of the eROSITA instrument [11] we simulated the event distribution of one of the ecliptic polar fields with a diameter of $5^{\circ}$ after the 4 year eROSITA survey (see Figure 4). Only AGN with a flux below $10^{-11} \mathrm{erg} \mathrm{s}^{-1} \mathrm{~cm}^{-2}$ were included to avoid the effects of bright sources dominating the field of 


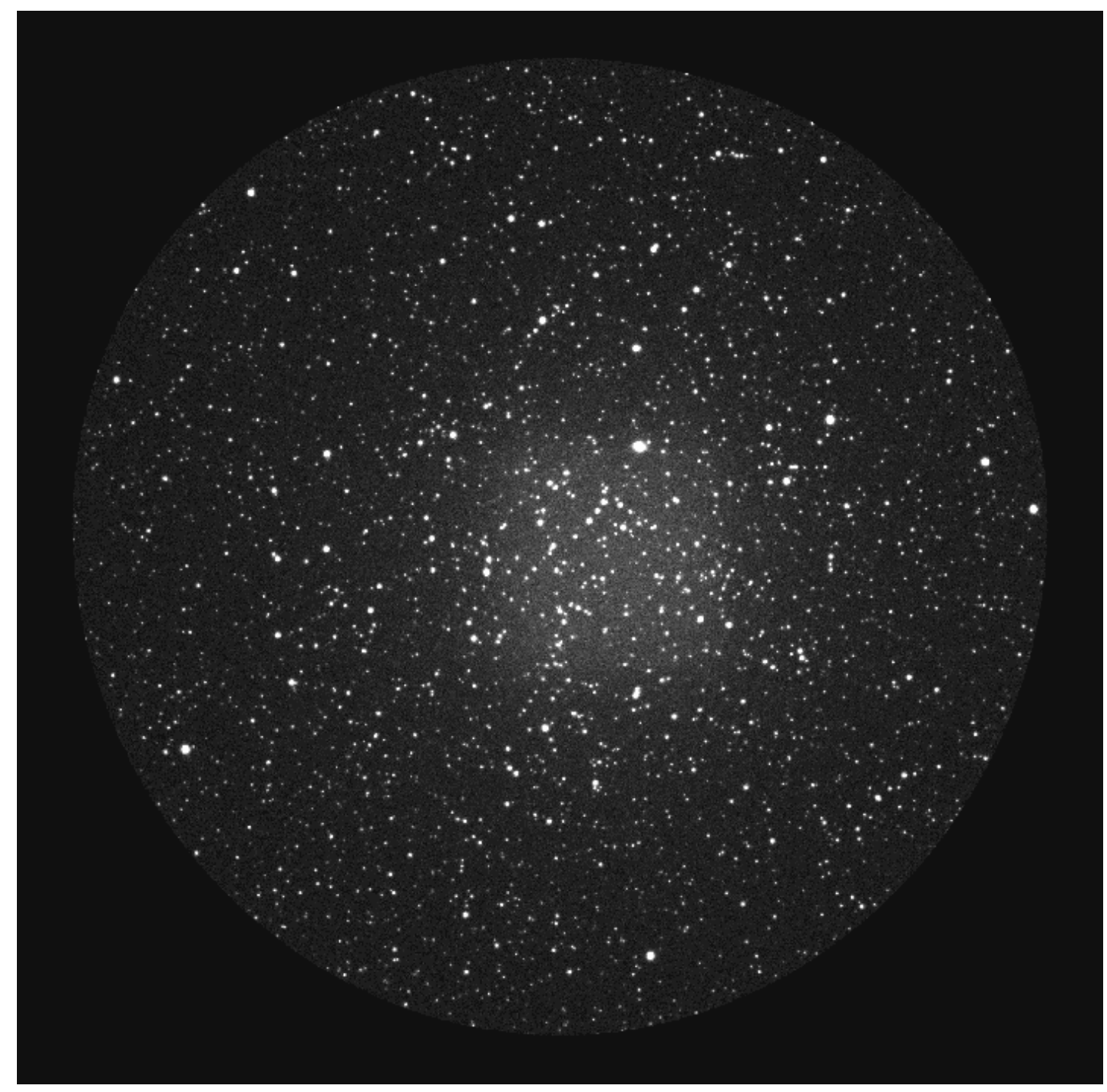

Figure 4: Simulation of a full 4 year survey polar field of AGNs

view. The diffuse emission at the center of the polar field is caused by a higher number of background events due to the deep exposure of that area.

The observation has been simulated with the Monte Carlo simulation toolkit SIXTE (SImulation of X-ray TElescopes, [11]). This software comprises a model of the eROSITA instrument implementing specific features such as an angle- and energy-dependent PSF model and vignetting function resembling the imaging properties and the pixel size, CCD operation, and energy resolution of the detector. The setup of the instrument is based on calibration files ensuring the physical accuracy of the implemented model.

Based on a SIMPUT source catalog, individual photons are produced and processed through the instrument model in order to obtain an event file [10]. The software allows to simulate an observation in the slew mode typical for the eROSITA all-sky survey. 


\section{Acknowledgments}

This research was funded by the Bundesministerium für Wirtschaft und Technologie under Deutsches Zentrum für Luft- und Raumfahrt grant number 50 QR 0801 and 50 OO 1111.

\section{References}

[1] Brand, T., 2011, Bachelor's thesis, Friedrich-Alexander University

[2] Gilli, R., Comastri, A., \& Hasinger, G. 2007, A\&A, 463, 79

[3] Kolodzig, A., Gilfanov, M., Sunyaev, R., et al. 2012, ArXiv e-prints

[4] Merloni, A., Predehl, P., Becker, W., et al. 2012, ArXiv e-prints

[5] Predehl, P., Hasinger, G., Böhringer, H., et al. ,in Space Telescopes and Instrumentation II: Ultraviolet to Gamma Ray, ed. M. J. L. Turner, G. Hasinger, Vol. 6266, Proc. SPIE, OP

[6] Predehl, P., Andritschke, R., Bornemann, W., et al. 2007, in UV, X-Ray, and Gamma-Ray Space Instrumentation for Astronomy XV, ed. O. H. W. Siegmund, Vol. 6686, Proc. SPIE, 668617

[7] Predehl, P., Andritschke, R., Böhringer, H., et al. 2010, Space Telescopes and Instrumentation 2010: Ultraviolet to Gamma Ray, ed. M. Arnaud, S. S. Murray, T. Takahashi, Vol. 7732, Proc. SPIE, 77320U

[8] Predehl, P., Andritschke, R., Becker, W., et al. 2011, in UV, X-Ray, and Gamma-Ray Space Instrumentation for Astronomy XVII, ed. O. H. Siegmund, Vol. 8145, Proc. SPIE, SPIE), 81450D

[9] Predehl, P., 2012, in Space Telescopes and Instrumentation 2012: Ultraviolet to Gamma Ray, Vol. 8443, Proc. SPIE, 84431R

[10] Schmid, C., Martin, M., Wilms, J., et al. 2010, in X-ray Astronomy 2009; Present Status, Multi-Wavelength Approach and Future Perspectives, Vol. 1248, 591

[11] Schmid, C., 2012, Ph.D. thesis, Friedrich-Alexander University

[12] Ueda, Y., Akiyama, M., Ohta, K., \& Miyaji, T. 2003, ApJ, 598, 886 